\title{
THE DETERMINATION OF VANADIUM IN VANADIUM AND CHROME-VANADIUM STEELS
}

\author{
By J. R. Cain
}

CONTENTS

I. Introduction.

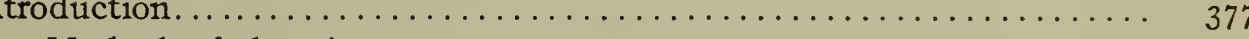

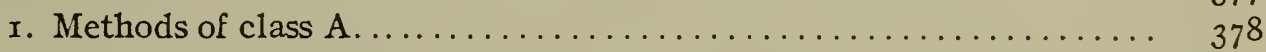

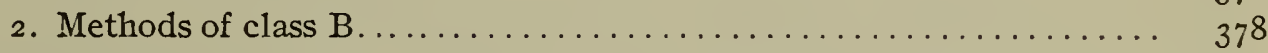

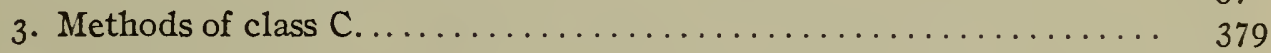

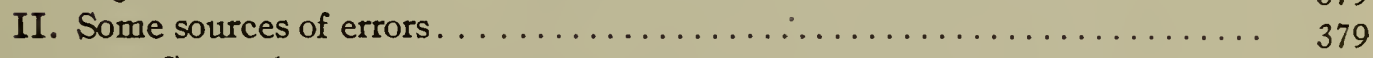

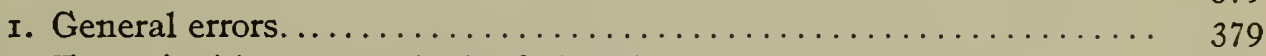

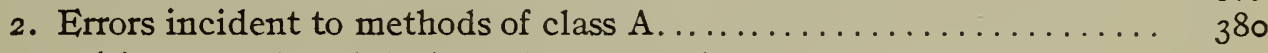

(a) Iron reduced during ether extraction. .............. $3^{80}$

(b) Action of sulphuric acid on organic substances........... 380

(c) Too early addition of sulphuric acid............... 380

(d) Too prolonged evaporation with sulphuric acid.......... 380

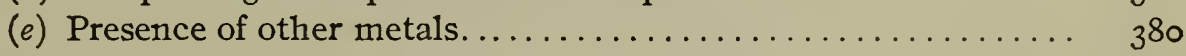

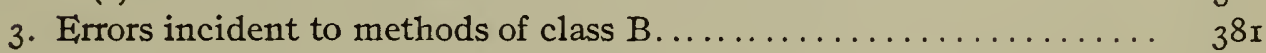

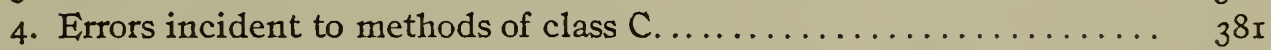

III. Preliminary work toward a new method for vanadium . . . . . . . . . $33_{3}$

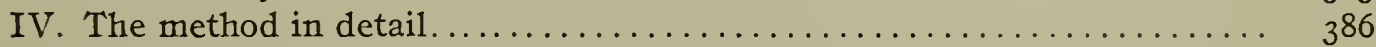

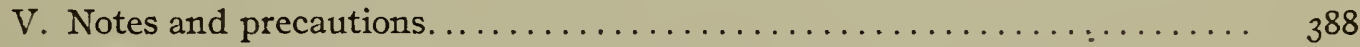

VI. Analytical data................................. $39 . \ldots \ldots$

VII. Summary . . . . . . . . . . . . . . . . . . . . . . . . . 392

\section{INTRODUCTION}

Vanadium has been called by metallurgists a "homeopathic" remedy because of the remarkable results obtained by small additions of it to other metals, notably to steel. Carbon steels or alloy steels as produced in this country when they carry vanadium usually contain from o. Io per cent to $\mathrm{I} .00$ per cent. It has been claimed by some that the element acts by removing dissolved gases, especially nitrogen, from the molten steel, with resulting elimination in the slag of the greater part added, and that the amount left in the steel itself is of secondary importance. However, others have 
shown that the microscopic structure of steel is greatly modified by the residual vanadium in the amounts in question here, and, as might be expected, there are corresponding changes in physical properties. Hence it will be seen that a high degree of accuracy in determining vanadium in steel is to be desired. Considerations such as these, combined with requests from many sources, led the Bureau of Standards to undertake the preparation of a vanadium standard steel similar to the analyzed plain carbon steels which it has issued for some time. In conformity with the usual practice of the bureau, samples were analyzed by its chemists and by prominent technical and works chemists (eleven in all). The first figures thus obtained for vanadium were so unexpectedly discordant as to indicate sources of error in methods used which had probably been overlooked or unrecognized. Accordingly, some of the most probable of these were investigated by the bureau. The results of this investigation were communicated to those participating in the cooperative work and a satisfactory concordance in the final vanadium figures was very soon obtained. Inasmuch as some of these sources of error do not seem to have been sufficiently emphasized in the literature, concise statements regarding them may be of service. Three classes of methods were used by the cooperating analysts:

\section{CLASS A}

Methods like that first described by Campagne ${ }^{1}$ and found with little variation from the original in many textbooks on steel analysis. The hydrochloric acid (ferric) solution of the steel is extracted with ether, which removes most of the iron, leaving the vanadium in the aqueous layer. This portion is evaporated to dryness and the operation repeated two or three times with fresh portions of hydrochloric acid. The latter reduces the vanadium to the quadrivalent state; the final evaporation is made with sulphuric acid and is continued until fumes are given off strongly. The solution is then titrated against permanganate.

\section{CLASS B}

Methods depending upon the reduction of quinquivalent to quadrivalent vanadium by ferrous sulphate, either by titrating 
directly against a ferrous solution or adding excess of the same and titrating back with bichromate; potassium ferricyanide is used either as an external indicator or by adding it directly to the solution undergoing titration.

\section{CLASS C}

Most of the iron is extracted as under class A, the hydrochloric acid replaced by nitric acid and the vanadium separated from iron, chromium, etc., by pouring into a boiling solution of sodium hydroxide. The vanadium goes into the filtrate, and is precipitated by mercurous or lead salts, the final estimation being either gravimetric or volumetric.

It seems likely that these three classes of methods comprise those most used in this country, inasmuch as the group of cooperating chemists was typical. The same methods are used with little or no change for the analysis of chrome-vanadium steels carrying usually from 0.2 per cent to 5 per cent chromium.

\section{SOME SOURCES OF ERROR}

\section{GENERAL ERRORS}

An error in all methods where final titration is made against permanganate may arise by failure to deduct the blank caused by the presence of elements other than vanadium; this may be particularly high in such methods as that of Campagne where, if the ether extraction is not carefully done, there may be large amounts of ferric sulphate present during the titration. Campagne himself speaks (loc. cit.) of this point, but it is not usually mentioned in textbooks. If salts yielding green or blue solutions are present, these may obscure the true end point, requiring several tenths of a cubic centimeter of tenth normal permanganate in excess. Indeed, in low vanadium products the blank may be much larger than the amount required by the vanadium, or if the analyst does not make a qualitative test for vanadium he may report its presence when the steel contains none. Another point to be observed is the temperature of the solution being titrated. The optimum temperature is $70^{\circ}$ to $80^{\circ} \mathrm{C} .^{2}$ It is almost impossible

${ }^{2}$ Hillebrand: Analysis of Silicate and Carbonate Rocks, Bull. 422, p. I5I. U.S. Geol. Survey. 
to secure a good end point in a cold solution. On the other hand, the work of Sarkar and Dutta ${ }^{3}$ and of others on the reduction of permanganate by hot sulphuric-acid solutions of manganous sulphate shows the importance of not titrating at too high a temperature.

\section{ERRORS INCIDENT TO METHODS OF CLASS A}

(a) Iron reduced during ether extraction.-During the ether extraction there is almost always some iron reduced to the ferrous condition; this is not always reoxidized during the course of the analysis before the titration and therefore might be calculated as vanadium if this fact is not taken into consideration.

(b) Action of sulphuric acid on organic substances.-The ether, or impurities in it, may act on the strong sulphuric acid when the solution is evaporated to small volume with this acid. There are then present substances which may act on permanganate. In this laboratory separation of carbonaceous material has been observed at this stage, with accompanying odor of sulphur dioxide.

(c) Too early addition of sulphuric acid.-The addition of sulphuric acid before the solution has been repeatedly evaporated with fresh portions of hydrochloric acid is to be avoided, because, if the sulphuric acid is added before reduction of vanadium to the quadrivalent state has been attained, the reduction is likely to be incomplete.

(d) Too prolonged evaporation with sulphuric acid.-If the evaporation with strong sulphuric acid is too prolonged or carried out at too high a temperature the reverse reaction, resulting in the oxidation of vanadium tetroxide to vanadium pentoxide by the sulphur trioxide, may take place. ${ }^{4}$

(e) Presence of other metals.-No other metals capable of oxidation to higher valence by permanganate should be present. Those most likely to be encountered in steel analysis are chromium, molybdenum, and tungsten. The latter two may be removed before titration without great difficulty; the methods hitherto given for separating vanadium and chromium are so laborious that this

${ }^{3}$ Zs. Anorg. Chem., 67, p. 225; 1910.

${ }^{4}$ Koppel and Behrendt: Zts. Anorg. Chem., 35, p. 156; 1903. 
precaution is often omitted in the case of chromium. A correction for the latter is invariably necessary, particularly in some steels where the chromium and vanadium may be present in the ratio of twenty or forty to one. Moreover, the amount of chromium oxidized to chromic acid is greater when titration is made at $70^{\circ}$ to $80^{\circ}$, as is imperative for a good vanadium end point than where the solution is titrated cold, as is sometimes recommended. (For details regarding this correction for chromium, see "Analysis of Silicate and Carbonate Rocks," Hillebrand, Bull. 422, pp. I 52-I 54, U. S. Geol. Survey.)

\section{ERRORS INCIDENT TO METHODS OF CLASS B}

Methods requiring the use of ferricyanide as indicator for ferrous salts when quadrivalent vanadium and ferrous salts are also present, must of necessity be very uncertain, yet these are the conditions under which the determination is carried out. The reason for this is very evident, when it is remembered that ferricyanide in acid solution rapidly oxidizes quadrivalent vanadium to the quinquivalent condition with resulting production of ferrocyanide. The latter reacts at once with the ferric iron, so that a blue color is present in the drops of indicator as soon as an appreciable amount of vanadium is reduced and long before the color due to excess of the titrating solution makes its appearance. Consequently such methods, if at all applicable, must yield results varying with the operator and requiring arbitrary and uncertain correction factors. Much difficulty was experienced in attempting to determine vanadium in pure vanadium solutions by this method; the reason for this, as stated above, was soon discovered, and later references to the same matter were found in the literature. $^{5}$

\section{ERRORS INCIDENT TO METHODS OF CLASS C}

Vanadium is almost always carried down by the precipitate of ferric and chromic hydroxides, etc., upon pouring into sodium hydroxide. Usually two or three precipitations are necessary to obtain all the vanadium in the alkaline filtrate. The difficulty

${ }^{5}$ Campagne, loc cit. Brearley and Ibbotson: Analysis of Steel Works' Materials, p. 89 . 
seems to be largely due to the presence of manganese, which, of course, the ether does not extract, for by working in the absence of manganese it has been found possible to make good separations with one precipitation. However, there is no simple method for removing manganese at this stage of a vanadium determination in steel without introducing other complications. If chromium is present another difficulty is added, for part of the manganese is oxidized by the air and precipitated while the sodium hydroxide solution is being boiled to secure complete precipitation of chromium and complete extraction of vanadium from the precipitated hydroxides; this peroxide rapidly oxidizes some of the chromium to chromate, which goes into the filtrate with the vanadium. If the sodium hydroxide precipitation is repeated, or a third precipitation is necessary, as may happen, a very large amount of chromium goes into the filtrate. In fact, the first operation leaves enough chromium with the vanadium to introduce serious error in determining the latter unless a correction is made; furthermore, the same cause may require appreciable correction to be applied to the chromium. The addition of sulphurous acid to the acid solution of the steel just before it is poured into the alkali, as is recommended sometimes, does not help, for, of course, it is not effective after the solution is strongly alkaline. Another source of trouble is the organic matter mentioned above as sometimes coming from ether; this causes appreciable amounts of iron and chromium to dissolve in the filtrate with the vanadium. It is evident that all of the sources of difficulty, except the last mentioned, are present in even greater degree when an attempt is made to carry out the caustic soda separation without a preliminary extraction of most of the iron by ether. Thus it will be seen that to separate the vanadium from the iron and to obtain all of it in the filtrate may be, and usually is, a long and complicated operation. When this is accomplished there may be present a large amount of chromium, and this occasions another series of difficulties, for the chromate precipitates with the vanadate, whatever the method of precipitation. The only alternative is to titrate the vanadium in presence of the chromium, making the uncertain corrections above mentioned, or to make an electrolytic separation as described later. 
When attempt is made to extract vanadium from steel by fusion of the oxides obtained by evaporating and baking a nitric acid solution of the metal, an old method which is now probably little used for this class of material, the same series of difficulties as enumerated under class $\mathrm{C}$ is encountered. It seems almost impracticable to extract quantitatively, say, one-tenth of I per cent of vanadium in this manner without undue expenditure of time and labor.

It is clear, then, that there are marked defects in the most commonly used methods for determining vanadium in steel, unless the analysis is conducted with extreme care, requiring more time than is usually available in a technical laboratory, or unless the analyst is so experienced that he can recognize and correct for disturbing factors. Accordingly, the present research was started with the hope that a simpler and more accurate procedure might be outlined, and one which would at the same time be reasonably short.

\section{PRELIMINARY WORK TOWARD A NEW METHOD FOR VANADIUM}

Much work was done with the idea of determining vanadium without a preliminary separation from iron. It seemed possible that the vanadium might be oxidized to vanadate and then reduced by hydrobromic acid by distilling in an apparatus suitable for collecting the liberated bromine in alkali. The difficulty, however, is to secure oxidation of the vanadium and then get rid of the excess of oxidizer without at the same time reducing some vanadic acid. It was found that the vanadium of a dilute sulphuric acid solution of a vanadium or chrome-vanadium steel could be oxidized easily enough in the cold by manganese dioxide without converting any noticeable amount of chromium to chromate, the excess of manganese dioxide being eliminated by filtration. But when such a solution was placed in the distilling apparatus and distilled after the addition of a large excess of hydrochloric acid and potássium bromide, as recommended by Edgar, ${ }^{6}$ there was almost always more bromine liberated than corresponded to the vanadium present. Many efforts were made to get rid of the disturbance, such as long boiling of the oxidized steel solution before placing in the distilling apparatus, reduction of the amount of suphuric acid

${ }^{6}$ Am. Jour. Sci., 27, p. 174; 1909. 
used for solution to the minimum necessary, etc., but while the results were usually as accurate as those obtained by any of the methods above described, there were occasional and unexplained irregularities which finally led to the abandonment of the method for technical purposes. This is regrettable, for, theoretically, such a procedure is ideal; practically it would be extremely short and simple. It seems likely that the trouble experienced was due either to the known solubility of manganese dioxide in sulphuric acid or to the action of sulphuric acid itself on the hydrobromic acid in the strongly acidified solution in the distilling flask.

From this it appeared desirable to develop a method which would obtain the vanadium in a solution free from all other metals, so that one might always be certain that the oxidizing or reducing action, whichever is made the basis of a volumetric method, is exerted by vanadium compounds and nothing else. The manner of accomplishing this was suggested in part by a dissertation of Albert Steffan, ${ }^{7}$ who used barium carbonate for precipitating vanadium and chromium, together with relatively little iron, from the (ferrous) solution of the steel. The principle at the base of the method has long been applied to other separations. It has invariably been recommended until recently to conduct this precipitation in the cold, shaking frequently and allowing to stand for many hours. It has been found, however, by numerous experiments here, that a few minutes boiling with the carbonate will completely precipitate much larger amounts of vanadium and chromium than are likely to be encountered in steel analysis; furthermore, the precipitates are free from manganese, a distinct advantage from many standprints over the ether separation. The shortening of the time required for precipitation when solutions are boiled has recently been noted by others in connection with steel analyses; also the use of zinc oxide as a precipitant. ${ }^{8}$ The precipitate obtained in this way contains the insoluble matter from the solution of the steel, a little iron, all the chromium and vanadium, and a considerable excess of the precipitant. The vanadium and chromium may be extracted by fusion with sodium carbonate, but

${ }^{7}$ Ucber die Bestimmung von Kleinen Mengen an Chrom und Vanadin in Gesteinen und Stahlarten, Zürich, r 902 .

${ }^{8}$ Slavik, Chem. Ztg., 34, p. 648; r 9 ro. 
the barium holds vanadium tenaciously and more than one fusion is usually necessary, so that this way of handling the precipitate, although apparently simpler, is longer than the one to be described later.

Smith ${ }^{9}$ describes an electrolytic method for separating vanadium from iron by driving the latter into a mercury cathode, and in another section he gives directions for electrolytically precipitating chromium under practically the same conditions; hence it appeared feasible to separate both iron and chromium from vanadium by electrolysis when the three are together. Preliminary experiment showed this to be possible, and it was then decided to dissolve the precipitate mentioned above in appropriate manner and submit it to electrolysis in order to obtain a pure vanadium solution. It seemed desirable, however, to be rid of the excess of the precipitant before electrolyzing. The necessary excess being relatively large, it would take too long to drive the iron and chromium out of solution if the precipitant were to accompany them into solution and be deposited along with them, as would be the case with zinc oxide, for instance. Moreover, it seemed desirable to dissolve the steel in sulphuric acid, as the electrolytic deposition is made from sulphate solution. This, of course, made inconvenient the use of barium carbonate as precipitant. It was therefore decided to try the suitability of cadmium carbonate for the purpose, with the idea of throwing out the excess of cadmium by hydrogen sulphide from the acid solution of the carbonate precipitate. This method gave entirely satisfactory results. The rate of precipitation of the vanadium and chromium seems even more rapid than with zinc oxide or barium carbonate. The precipitate of cadmium sulphide obtained in a boiling, very slightly acid solution is easily filtered and never carries down vanadium.

The precipitation of vanadium and chromium from a reduced and boiling solution of steel by any of the precipitants herein mentioned succeeds equally well in hydrochloric or sulphuric solutions, but seems to proceed a little faster with cadmium carbonate and a sulphate solution.

${ }^{9}$ Electroanalysis, fourth edition, p. 258. 


\section{THE METHOD IN DETAIL}

Dissolve 2 to 4 grams of steel in 40 to $60 \mathrm{cc}$ of ro per cent (by volume) sulphuric acid in a covered $300 \mathrm{cc}$ Erlenmeyer flask. Filter off the insoluble, wash two or three times with water, ignite and fuse for a few minutes with acid potassium sulphate, adding the aqueous solution of the fusion to the main solution. If the steel will not dissolve readily in sulphuric acid, hydrochloric acid may be used, fusing the insoluble as before. ${ }^{10}$ Nearly neutralize the solution of the steel with saturated sodium carbonate solution. Then add finely pulverized cadmium carbonate in small portions at intervals of four or five minutes, boiling vigorously between times, keeping the flask well covered. A gram or two of carbonate should remain undissolved at the end of the operation. About fifteen or twenty minutes' boiling always suffices. The time may be even shorter for vanadium steels containing little or no chromium. Allow the precipitate to settle and filter rapidly, so as to prevent oxidation and precipitation of iron on the filter (an I I cm S. and S. No. 589 white-label filter is recommended). Wash the precipitate twice with hot water; no care need be taken to remove all of it from the flask. Dissolve the precipitate with the minimum of nearly boiling io per cent sulphuric acid, catching the filtrate in the flask. Boil to insure solution of material adhering to the sides of the flask. Cool and nearly neutralize with ammonia; there should be no more free acid present than is necessary to prevent the iron from precipitating by hydrolysis when the solution is boiled. Pass a rapid current of hydrogen sulphide for a few minutes while the solution is boiling vigorously. Let the precipitate settle, filter it off, and wash two or three times with hot water. Concentrate the filtrate if necessary and electrolyze in a volume of 60 to $70 \mathrm{cc}$, using 5 to 6 amperes at 6 to 7 volts. The amount of mercury used in the special apparatus described below was about 200 grams. The solution is tested for iron by ferricyanide; usually when no iron test is obtained all the chromium is removed. However, should

\footnotetext{
${ }^{10}$ In this case the carbonate precipitate must be dissolved in sulphuric acid and the separation repeated in sulphate solution, for the precipitate first obtained contains enough chloride to give trouble if dissolved in sulphuric acid and directly electrolyzed.
} 
there be an unusually large amount of chromium relatively to the iron, this might not happen. In this case the test should be made by removing three or four cubic centimeters of the solution, adding a few drops of hydrogen peroxide and boiling for a few minutes after the brown color, due to vanadium peroxide, disappears. If the solution now remains clear and colorless on adding ammonia, the electrolysis is ended. Acidify the test portion with sulphuric acid before returning it to the electrolyzing apparatus. The simpler ferricyanide test usually answers for all purposes; the complications mentioned earlier, due to reduction of ferricyanide by quadrivalent vanadium, do not interfere in this case, inasmuch as there is no ferric iron to react with the resulting ferrocyanide. When the solution no longer gives a test for iron or chromium, remove it from the apparatus and wash the mercury two or three times with 25 or 30 cc of water while the current is still passing. Add two or three cubic centimeters of sulphuric acid ( $I-I$, by volume), heat to $70^{\circ}$ or $80^{\circ} \mathrm{C}$, and add permanganate from a pipette until there is a strong pink color. Run sulphur dioxide into the boiling solution for a few minutes; then pass a rapid current of carbon dioxide until the escaping steam gives no test for sulphur dioxide. Filter, preferably through asbestos, ${ }^{11}$ or a Munroe crucible, cool the solution to $70^{\circ}$ or $80^{\circ} \mathrm{C}$, and titrate against $\frac{\mathrm{N}}{5 \mathrm{O}}$ potassium permanganate. For extreme accuracy repetition of the reduction and titration is recommended. The reduction may also be made by hydrogen sulphide, if desired. ${ }^{12}$ With special facilities a determination may be completed in one and one-half hours, or less.

11 The result will be slightly high if a paper filter is used. This is due to the hydrolyzing and solvent action of the acid solution on the paper (even the best washed filter paper). With alkaline solutions very serious errors may be incurred from ignorance of this fact.

12 Bleecker, Met. and Chem. Eng., 9, p. 209, Apr., Igri, reduces vanadate solutions electrolytically, using a platinum dish as cathode. This method appears short and simple and might be adopted in many cases. No work has been done here, however, along this line and the exact conditions for complete reduction to the hypovanadate stage, and no further, would probably have to be determined. It is not safe to titrate directly the solution obtained by electrolyzing as described above without the preliminary permanganate oxidation followed by reduction and filtration; to do so usually gives irregular results. 


\section{NOTES AND PRECAUTIONS}

Sufficient exclusion of air is secured by dissolving the steel in a flask kept well covered by a watch glass. The fusion of the insoluble is absolutely necessary, as a large proportion of vanadium remains here; indeed, Nicolardot has proposed methods whereby, with complete exclusion of air, it is possible to secure practically all the vanadium in the insoluble portion when operating on certain classes of materials. This makes a quick and easy qualitative test for vanadium in steel, for as soon as the carbon is burned off the characteristic appearance of the fused vanadium pentoxide is very striking.

The treatment of the insoluble requires but a few minutes. The operations of solution, of cadmium precipitation, and of filtration must be done promptly, for a delay between solution and precipitation gives opportunity for oxidation of iron. This is to be avoided, since it increases the amount of iron going into the carbonate precipitate, thereby prolonging the electrolysis and also interfering with the complete precipitation of the vanadium and chromium. In this latter connection it seems that the particles of carbonate become rapidiy coated with the excessive precipitate of ferric hydroxide, and this interferes with their efficiency in neutralizing the solution. The operations of filtering and washing must be particularly rapid. There is no difficulty here if the solution, after boiling, is allowed to stand a few minutes before filtering; the precipitate settles readily and is easily filtered. Two washings with hot water are sufficient. Sometimes a little difficulty is experienced in dissolving the precipitate completely from the filter. This is particularly true if the filtration and washing have not been done rapidly and promptly, giving opportunity for a difficultly soluble coating to form over the surface, due to oxidation and precipitation of iron. However, this trouble will never be serious if the operations are conducted as directed; moreover, even if this iron is not all removed from the filter it is never found to contain vanadium after two or three washings with the dilute acid. The advantage of a carbonate precipitant over an oxide precipitant is shown here, because the carbon dioxide evolved during the process of solution very effectually breaks up the diffi- 
cultly soluble surface layer, permitting the acid to get at the main body of the precipitate. On the other hand, when using zinc oxide it is often very difficult to dissolve the precipitate with dilute sulphuric acid. The hydrogen sulphide precipitation of the excess of cadmium is accomplished quickly; the solution should be nearly neutralized with ammonia; sodium hydroxide or carbonate should not be used, as in this case sodium would be driven into the mercury during electrolysis, not only prolonging this operation, but introducing other complications. If the degree of acidity is right and a rapid current of hydrogen sulphide is used, the solution being boiled vigorously while this is passing, the cadmium sulphide settles readily and is easy to filter and wash. The solution after filtration can be transferred at once to the electrolytic apparatus, which may have any of the usual forms, such as the convenient small beakers with sealed-in platinum wires at the bottom, described by Smith. ${ }^{13}$ If many determinations have to be made, however, the apparatus shown in Fig. I has been found very convenient.

The apparatus is a separatory funnel, shown in half size in Fig. I, with an inwardly projecting tube $A$. The bore of the stopcock should preferably be as

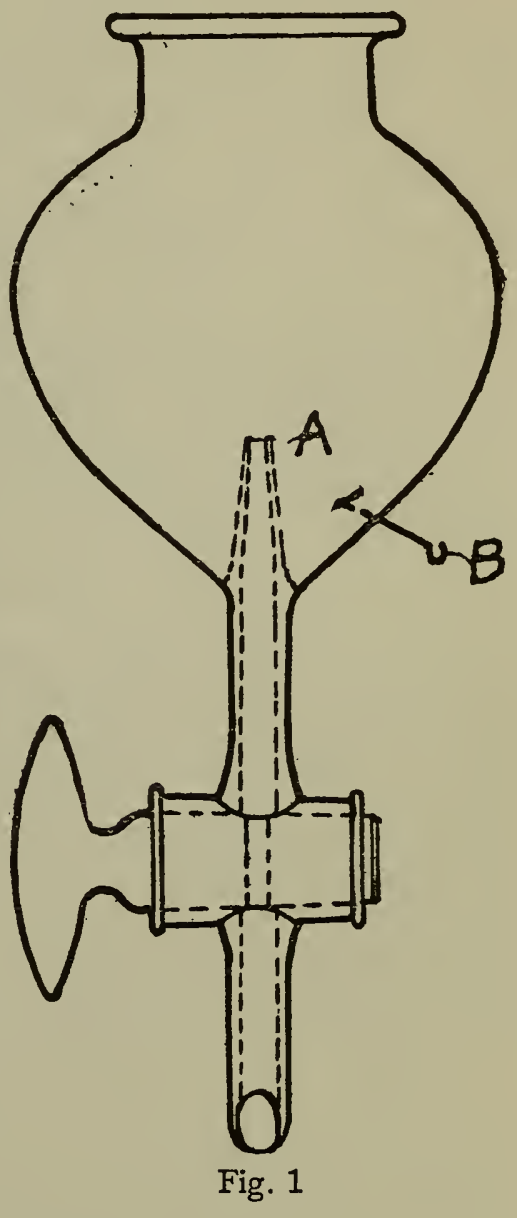
large as shown. A No. 18 platinum wire is sealed in at B. The apparatus is filled with mercury to within $\mathrm{I}$ or $2 \mathrm{~mm}$ of the end of tube A, which itself is completely filled with mercury, and electrical connection is made to the negative terminal of the battery. The anode may have any of the usual rotating forms. The electrolyzing vessel is conveniently supported by an iron ring clamped to the stand carrying the motor for 
rotating the anode. When electrolysis is completed, the anode is brought as close as possible to the surface of the mercury without short-circuiting and the electrolyte is allowed to run out by opening the stopcock. The washing is done very easily by a jet of water from a wash bottle, leaving the stopcock open during the operation, the course of the washing being followed by the ammeter.

If a rotating anode is used, the electrolysis is accomplished in fifteen or twenty minutes. The mercury may be used over and over again without purification. This is another advantage of removing the excess of precipitant before electrolysis, for the amount of foreign material going into it is thus reduced to a minimum. When a stock of mercury saturated with iron and chromium has accumulated it may be purified by the rapid method of Hildebrand..$^{14}$ A fair degree of purification can be attained in a few minutes by shaking in a separatory funnel with concentrated hydrochloric acid.

\section{ANALYTICAL DATA}

Table I gives some results that have been obtained on synthetic mixtures of chromium, vanadium, and iron. The vanadium was added from a stock solution of sodium vanadate which was carefully standardized by reducing several portions with sulphur dioxide and titrating against permanganate; the iron was from a sulphuric-acid solution of a Bessemer steel with o. I per cent carbon, and the chromium from a roughly standardized chrome-alum solution. Precipitation by cadmium carbonate was made in the presence of 4 grams of iron, one-half of the solution of the precipitate being used for electrolyzing.

${ }^{14}$ Jour. Am. Chem. Soc., 31, p. 933; r909. 
TABLE I

\begin{tabular}{c|r|r|r|r}
\hline \multirow{2}{*}{ Number } & \multicolumn{3}{|c|}{ Vanadium } & \multirow{2}{*}{ Chromium } \\
\cline { 2 - 3 } & Present & \multicolumn{1}{|c}{ Found } & \multicolumn{1}{c}{ Error } & \\
\cline { 2 - 3 } 1 & 0.0030 & 0.0030 & 0.0000 & 0.0286 \\
2 & .0030 & .0030 & .0000 & .0572 \\
3 & .0101 & .0102 & +.0001 & .0358 \\
4 & .0117 & .0116 & -.0001 & .0286 \\
5 & .0199 & .0204 & +.0005 & .0572 \\
6 & .0376 & .0371 & -.0005 & .3150 \\
7 & .0407 & .0410 & +.0003 & .3150 \\
$* 8$ & .0010 & .0010 & .0000 & None \\
\hline
\end{tabular}

* In presence of 10 grams of iron.

Determinations were made on the vanadium steel standard of this bureau, first, from hydrochloric solution; second, from sulphuric solution. Duplicates gave 0.I45 per cent vanadium and 0.1 42 per cent vanadium from the hydrochloric solution. To these there had been added 0.0750 grams and 0.1064 grams chromium, respectively. In sulphuric solution there was obtained o.I45 per cent. The mean of several very careful determinations by bureau chemists, using different methods, was O.I 43 per cent. On the new chrome-vanadium standard now in preparation the method gives 0.203 per cent and 0.203 per cent. The averaged complete analysis of the vanadium standard and of the chrome-vanadium . standard (full data for the latter have not yet been received and the analysis given is not final) are given below:

TABLE II

\begin{tabular}{c|c|c|c|c|c|c|c|c|c|c}
\hline Standard & $\mathrm{C}$ & $\mathrm{Si}$ & $\mathrm{P}$ & $\mathrm{S}$ & $\mathrm{Mn}$ & $\mathrm{V}$ & $\mathrm{Cr}$ & $\mathrm{Ni}$ & $\mathrm{Cu}$ & $\mathrm{Mo}$ \\
\hline $\begin{array}{r}\text { Vanadium..... } \\
\text { Chrome - Vana- } \\
\text { dium......... }\end{array}$ &. .350 &. .303 & 0.035 & .0 .027 & 0.669 & 0.15 & 0.007 & 0.009 & 0.022 & 0.006 \\
\hline
\end{tabular}

The results show that a satisfactory degree of accuracy is attainable; this may be increased even more, if desired, by using a larger sample of the steel, for there is no reason why the vanadium should not be concentrated from Io grams or more of steel, if 
desired. The minimum amount of vanadium here determined, equivalent to o.or per cent in a steel (No. 8 of Table I), shows how delicate the method is; the maximum amount (No. 7 of Table I) shows the possibility of using cadmium precipitation, followed by electrolysis, for high vanadium products. The complete and quick precipitation of large amounts of chromium by cadmium carbonate is equally striking; a method for determining chromium in various kinds of steel, based upon these observations, is being worked out in this laboratory.

From what has been said of the effect of the presence of other substances when titrating hypovanadate solutions with permanganate it is evident that a method like this has distinct advantages in eliminating other metals. If the method is to be used for other steels than those for which it was devised, the effect of other metals must be considered. Such metals as copper and nickel would not interfere. The behavior of molybdenum and tungsten in appreciable amounts has not been investigated, but molybdenum, if at all precipitated by cadmium carbonate, may be eliminated during the hydrogen sulphide precipitation of the excess of cadmium. Tungsten might be removed by evaporation of the cadmium precipitate with nitric acid, followed by filtration. After conversion of the nitrate to a sulphate solution electrolysis could follow as above described. Titanium would probably be precipitated by the cadmium and remain with the vanadium through the electrolysis; however, it would not be reduced by sulphur dioxide or hydrogen sulphide. These points will be investigated as opportunity permits.

\section{SUMMARY}

I. Various errors in the usual methods for determining vanadium in steel are pointed out and in a few cases methods for correcting or eliminating these are indicated.

2. A new method based on precipitation of the vanadium by cadmium carbonate, followed by electrolysis, reduction, and titration, is described.

The writer is indebted to Dr. Hillebrand for many helpful suggestions during the course of this investigation.

Washington, April 24, igir. 